\title{
The Application of Ad Hoc Network in the Design of Helmet-type Information Platform for Earthquake Search and Rescue Missions
}

\author{
L.Y.ZHAO \\ ${ }^{1}$ China University of Geosciences Beijing 100083, China \\ ${ }^{2}$ National Earthquake Response Support Service, Beijing, 100049, China
}

W.J.HU*

National Earthquake Response Support Service, Beijing, 100049, China

M.DENG

China University of Geosciences Beijing 100083,China

Corresponding author: W.J.HU

ABSTRACT: The helmet-type information platform is used for the multi-source information acquisition and processing. Based on the AD HOC network and AODV protocol, the information from different devices and sensors transmits from rescuers to other members, such as the audio and video signal, the GPS, the poisonous gas sensors. With the network, the rescuers can connect staffs in one team or different teams, also can connect the control center. It provides more efficient collection and transmission of the field information, more accurate rescue decision and eventually improves the efficiency of rescuer.

Key words: AD HOC network; AODV protocol; helmet; Information transmit

\section{AD HOC NETWORK AND AODV PROTOCOL}

\subsection{Introduction of Ad Hoc network}

Ad Hoc is also called wireless Ad Hoc network which has been widely used in mobile video conference and emergency or temporary occasion in recent years. As the structure shown in Figure 1,there are three aspects advantages: dynamic topology, self-organizing structure and multi-hop routing (Mauz et al., 2009).

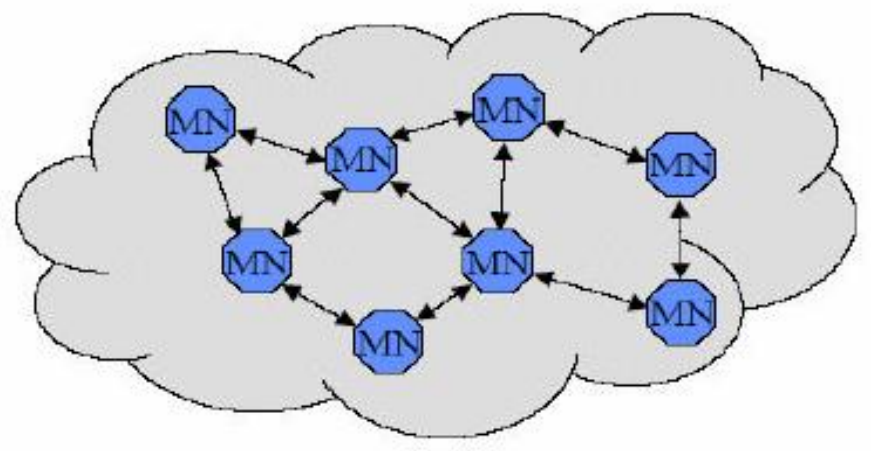

Figure 1. The structure of Ad Hoc.

(1)Dynamic topology. Take the helmet terminal for example, the rescuers may change their positions at any time and the number of the rescuers may alter suddenly, that makes the network topology change unpredictably. The Ad Hoc can keep the network from paralysis by adapting to these changes quickly.
(2) Self-organizing structure. Ad Hoc network does not need the base station or center node. All terminals in the network are equal and can be center node, also can join in or leave any time.

(3) Multi-hop routing. Since the communication distance is limited, a repeater is needed when a node wants to call another node while their distance is beyond its coverage. Any node in the network can be the route to actualize the relay and there could be more than one route to make sure the information arrive at the destination node. (Makoto et al., 2007).

These advantages of Ad Hoc assure the information network can be established rapidly, so the rescuers can connect staffs in one team or different teams, also can connect the control center as soon as they reach the designated area. With the network, the multi-source information may transmit from different terminals and control center.

\subsection{AODV protocol}

The protocol used in the helmet is the reactive routing protocol which needs less memory and power consumption. The routing table will be established and maintained during the session and then will be deleted when the session is ended. There are several circumstances for the start node $\mathrm{A}$ be connected with the target node B as shown in Figure2. 


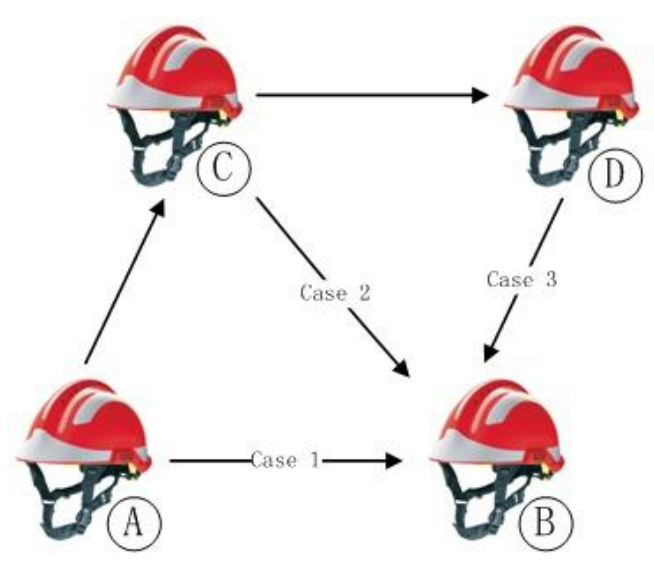

Figure 2. Multi-hop routing

In case 1 , node $A$ connect directly with the target node $\mathrm{B}$ and the AODV is not in use.

In case 2 , node $A$ is failed to call node $B$, so it sends RREQ message to all the nodes within its reach to find the best route, and after node A receive RREP message, the new routing table will be established (Perkins et al., 2010).In this case, node C connect directly with the target node $\mathrm{B}$, so the routing table in case 2 is node A,C,B.

In case 3 , node $A$ and node $C$ are both failed to call node $\mathrm{B}$ and there is node $\mathrm{D}$ connect directly with the target node B, so after node A send RREQ message and receive RREP message, the new routing table which is node A,C,D,B will be established.

However, there will be more rescuers than shown in Figure 2, so the routing procedure may be more complex in the missions, more than one relay nodes could be needed, and the topology may be more sophisticated.

\section{THE ARCHITECTURE OF HELMET-TYPE INFORMATION PLATFORM}

\subsection{Hardware structure of the helmet terminal}

As shown in figure 3 , the helmet terminal is composed of embedded main board, switch, gas sensor, camera, GPS module, vibrator, antenna, etc. Also there are wireless module, earphone and battery to finish the function of the terminal.

Considering the complex environment of disaster site, the helmet terminal is equipped with harmful gas sensor module, which can detect $\mathrm{CO}, \mathrm{SO} 2, \mathrm{CH} 4$, CL2 and other poisonous gases with changing the sensors. When the gas sensors detect the concentration of poisonous gas reached the threshold, the vibrator will vibrate and alarm, warning the rescuer to leave the dangerous area immediately. Electrochemical gas sensors are chosen as they have better performance than catalyst combustion gas sensors and semiconductor gas sensors, and usually electrochemical gas sensors have lower power consumption and can be safer in specific situations (Zhang et al., 2011).

Also in order to fix the location of both the rescuers and survivors in the earthquake rescue mission, GPS module is equipped. When survivor being finded, rescuers can report the information of survivor, such as the physical condition, the collapsed structure, the rescue members and equipments to the leader or expert to make the most optimized rescue strategy, if rescuers on the work site think they need help from the control center.

There are two power sources for the helmet terminal, the solar and lithium battery. A solar cell is embedded at the top of the helmet and works as main power, while the lithium battery which hanging in the waist or embedded into the clothes, designed as a backup power. With the calculation, the current of the helmet terminal can be limited under $400 \mathrm{~mA}$, considering the weight and the work time, we choose a lithium battery with the capacity of $6000 \mathrm{mAh}$ which can support the helmet terminal to work for more than 5 hours and the weight is 100 gram.

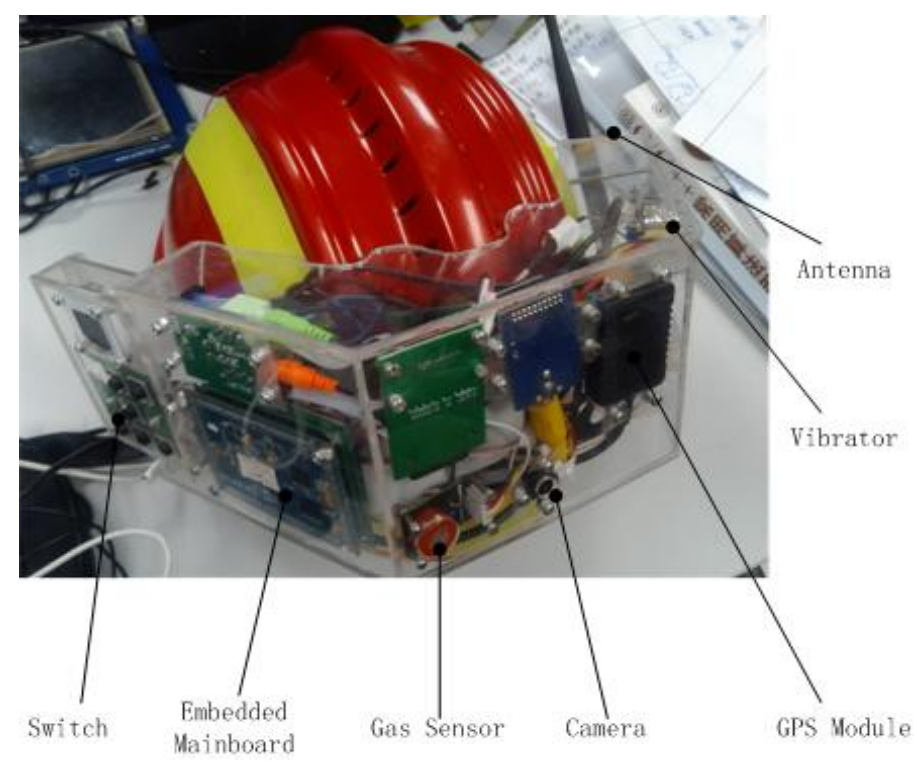

Figure 3.Prototype of the helmet

\subsection{Software structure of the helmet terminal}

Since there are many modules on the helmet terminal, the software needs to process data from all modules parallel to get synchronization, especially for the audio and video data. During the programming, nine thread that include the main thread are used to individually handle the tasks, such as the acquisition and transmission of data from the video, picture, GPS, and the acquisition, transmission, playing of the audio data. The software thread is shown as Figure 4. 


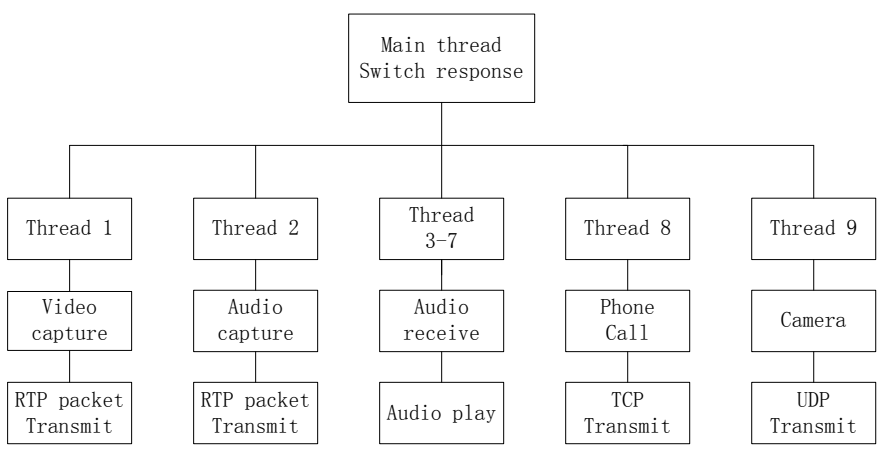

Figure 4. The flow chart of helmet terminal

\section{LAYOUT OF TEXT THE INFORMATION FLOW OF HELMET-TYPE INFORMATION PLATFORM}

\subsection{Summary}

Rescuers acquisite information using the devices and sensors on the helmet, such as the audio, video from the camera, the environment safety status from the gas sensors, etc. Then if the rescuers want to communicate with others members, the information could be transmitted to the target through different routes shown in Figure 5.

There could be three typical information transmission route in Figure 5 using respectively composed by AODV protocol, Wireless AP and Grid antenna. The first one is the information flow between rescuers in one work site. The second one is the information flow between rescuers in different work sites. The third one is the information flow between work site and control center.

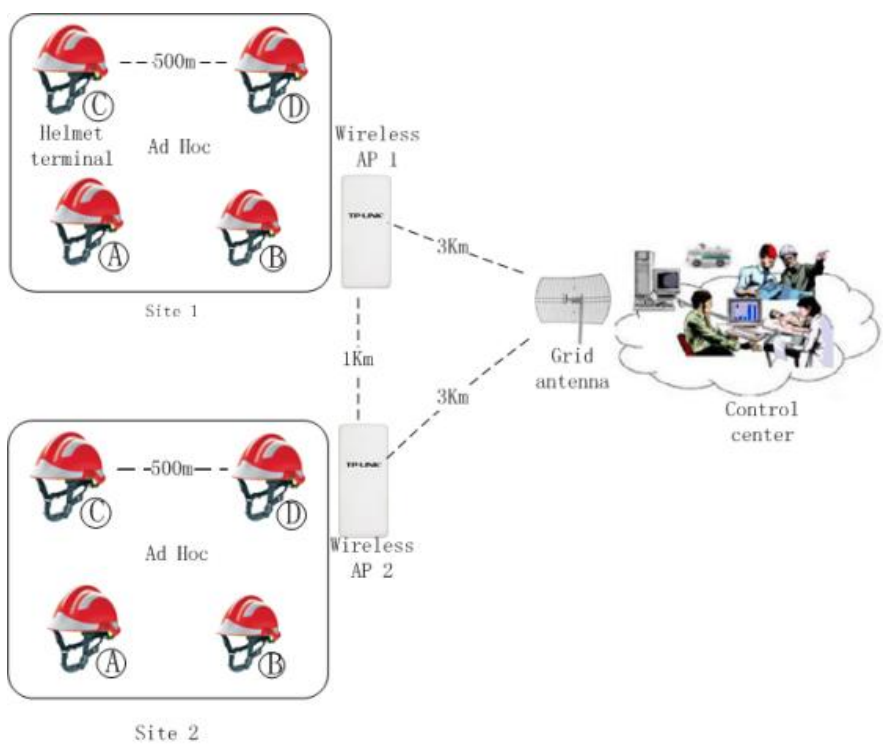

Figure 5.Information flow in the system

\subsection{Information flow in one work site}

The information flow between rescuers in one work site is the most basic pattern. As expatiated above in 1.2 ,there are three cases for the original rescuer to get connection with the target rescuer. The distance between two rescuers is designed to be 500 meters if the helmet terminals are directly connected.

When rescuer has data to transmit to an unknown target, the helmet terminal broadcasts a RREQ for the target. At each intermediate helmet terminal, a route to the rescuer is created when a RREQ is received. The receiving helmet terminal will rebroadcasts the RREQ if it is not the target and does not have a direct route to the target. In the contrary circumstances, the helmet terminal generates a RREP. The RREP is unicast in a hop-by-hop fashion to the source and every helmet terminal creates a route to the target with the RREP propagation. When the source helmet terminal receives the RREP, it recods the route to the target and can sending data. The source helmet terminal will choose the shortest hop count as the route when there are multi RREPs.

\subsection{Information flow in different work sites}

The information flow between rescuers in different work sites needs using the wireless AP, and the distance between two wireless APs is designed to be 1 kilometer if there is no other relay AP.

In figure 5, when rescuer in work site 1 wants to get connection with rescuer in other work site, the helmet terminal will work like below:

(1)The helmet terminal broadcasts a RREQ for the target and there will no RREP back in the area of work site 1 .

(2)The helmet terminal broadcasts a RREQ to the wireless AP 1 , then the wireless AP 1 will rebroadcasts the RREQ to other wireless APs in its coverage, such as wireless AP 2. The wireless AP 2 re-broadcasts the RREQ to other wireless APs and the helmet terminals in its coverage. In the same time, the route to the source helmet terminal is recoded.

(3)If one helmet terminal covered by the wireless AP 2 is the target, then the target helmet terminal will generates a RREP. The RREP propagates back to the source helmet terminal through the two wireless APs and the route is also recoded in order to keep the channel during the data transmission.

(4)Unluckly, if the target is not covered by the wireless AP 2, the other wireless AP need to search the helmet terminal in its coverage and re-broadcasts the RREQ to the wireless AP in its area. The procedure will sustained till the target helmet terminal is finded.

\subsection{Information flow between work site and control center}

The information flow between rescuers and the control center is the most complex pattern. This pattern uses the most components such as the helmet terminal, the wireless AP, the grid antenna. The distance between the wireless AP and the control 
center is designed to be 3 kilometers if there is no other relay AP.

In figure 5 , when rescuer in work site 1 wants to get connect with the control center, the helmet terminal will work like below:

(1)The helmet terminal broadcasts a RREQ to the wireless AP 1.

(2)If the wireless AP 1 has route to the control center, it will send the RREQ to the control center through the grid antenna. The RREP will be send back to the rescuer back through the channel. The channel will be keeped during the data transmission.

(3)If the wireless AP 1 does not have the route, it will re-broadcasts the RREQ to other wireless APs. The procedure will sustained till there is one wireless AP has the route to the control center.

\section{CONCLUSION}

Using the Ad Hoc network and AODV protocol, the information transmission between rescuers, disaster work site and the control center is actualized and there is the experiment to confirm the availability.

There are some aspects could be enhanced during the further research:

Improve the prototype of helmet terminal. The devices and sensors are equipped on the helmet, the better way is made them embedded in the helmet terminal to less the weight and volume, also the terminal would be good-looking.
The quality of audio and video will be affected by the environment such as wind, rain. How to using the hardware and software technology to keep the signal stable is also very important.

\section{ACKNOWLEDGEMENTS}

This work was supported in part by the National Science and Technology Support Project under Grant 2012BAK15B05.

\section{REFERENCES}

[1] Mauz, N., Amir, H., March 2009, Agent based Tools for Modeling and Simulation of Self-Organization in Peer-toPeer, Ad Hoc and other Complex Networks, Feature Issue, IEEE Communications Magazine, Vol.47 No.3, pp 163173.

[2] Makoto Ikeda, Giuseppe De Marco, Tao Yang, 2007,Leonard Barolli, Performance analysis of an ad hoc network for emergency and collaborative environments. 21st International Conference on Advanced Networking and Applications Niagara Falls, Canada, pp 21-23.

[3] Perkins, C., Belding-Royer, E., Das, S. 2010-06-18, Ad hoc On-Demand Distance Vector (AODV) Routing. IETF. RFC 3561. Retrieved.

[4] Zhang, C.Y., YU, T.Z. and Su, X.L. 2011 ,The working principle, performance and application of commonly used gas alarm, Chemical analysis and measurement, Chinese, pp70-71, Vol. 20, No. 4. 